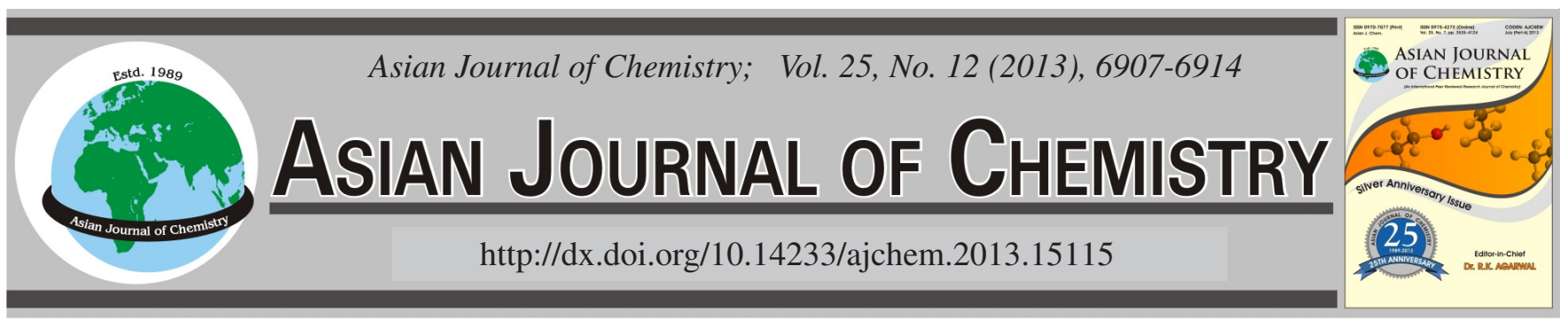

\title{
Computational Study on the Affinity of Flavonols Bonding with Collagen-Like Peptide
}

\author{
JiAHONG Lu ${ }^{1,2}$, PingPing Ding ${ }^{3}$, Wenhua Zhang ${ }^{2, *}$ and Bi Shi ${ }^{1}$
}

${ }^{1}$ National Engineering Laboratory for Clean Technology of Leather Manufacture, Sichuan University, Chengdu 610065, P.R. China
${ }^{2}$ Department of Biomass Chemistry and Engineering, Sichuan University, Chengdu 610065, P.R. China

${ }^{3}$ Department of Chemical Engineering, Sichuan University, Chengdu 610065, P.R. China

*Corresponding author: E-mail: zhangwh@scu.edu.cn

\begin{abstract}
The results of static experiments showed the affinity of the three flavonoids on the glutaraldehyde cross-linked collagen fiber followed the order:myricetin > quercetin > kaempferol. Molecular simulation revealed arginine of collagen-like peptide and phenolic hydroxyl in B ring of the flavonoids should be effective sites to dock and the difference in affinity of peptide to the flavonoids was consistent with the experimental results. Then bonding energies of well-docked complexes that calculated in water and ethanol showed ethanol could magnify the difference in affinity of collagen-like peptide to the three flavonoids. So if the ratio of solvents is properly adjusted, the three flavonoids may be separated. Further molecular dynamics simulations indicated the main mode of interaction of collagen-like peptide and flavonols was van der Wall force and H-bond, whereas electrostatic interaction contributes much less.
\end{abstract}

Key Words: Flavonol, Collagen-like peptide, Affinity, Molecular dynamics simulation.

\section{INTRODUCTION}

Myricetin, quercetin and kaempferol are polyphenol compounds that contain a C15 flavone skeleton (Fig. 1) and are widely distributed in plants, especially in fruits and vegetables $^{1}$. Their physiological activities such as anti-mutagenic and anti-carcinogenic effects in vitro and in vivo have been well documented $^{2-5}$. Myricetin has the antagonistic effect on platelet activing factor and the hypoglycemic effect ${ }^{6,7}$. Quercetin also exhibits antagonistic action for multi-carcinogen and restrains the growth of malignant turmors ${ }^{8}$. Kaempferol can reverse multidrug-resistance of tumor cell, protect the nerve cell and restrain protein kinase ${ }^{9}$. However, quercetin and kaempferol cause cell damage in the absence of oxidative stressors, but act beneficially in their presence ${ }^{10}$ and the content of the three flavonols in different plants is fairly different. Thus, it would be interesting to explore the possibility of isolating them from plant extracts in order to study further their medical effects. Our previous study indicated that an adsorbent made by collagen fiber exhibits excellent adsorption selectivity to flavonoid and alkaloid $^{11}$. However, the structure of myricetin, quercetin and kaempferol is very similar except for the number of phenolic hydroxyl group on B-ring. Then the understanding of the interaction and adsorption affinity between the three flavonols and collagen is the fundamental aspects of assessing the feasibility of an adsorption process for practical application.<smiles>COc1cc(-c2oc3cc(O)cc(O)c3c(=O)c2O)cc(O)c1-c1cc(O)c(O)c(O)c1</smiles><smiles>O=c1c(O)c(-c2ccc(O)cc2)oc2cc(O)cc(O)c12</smiles>

Kaempferol

Fig. 1. Structures of myricetin, quercetin, kaempferol 
Experimental findings proposed the interaction between collagen and polyphenols should be a synergistic effect of hydrophobic association and hydrogen bonding ${ }^{12}$. More detailed studies suggested that polyphenols interact preferentially with PRO, HPR and ARG side chains ${ }^{13,14}$. Due to the complexity of collagen system, it is difficult to identify the binding site and understand the interaction between collagen and ployphenol from the experimental point of view. Whereas, molecular simulation can be of help in illustrating experimental findings and predict the effect of experiment by using effective model ${ }^{15,16}$. Indeed, molecular mechanics and dynamics have been used to understand the interaction between collagen and gallic acid ${ }^{17}$. The hydroxyl, carboxyl and basic groups in the side chain of collagen has been considered as the potential sites to form hydrogen bonds with polyphenols $^{15-17}$.

In this paper, static experiments were carried out to obtain the adsorption affinity of the three flavonols on collagen fiber and molecular simulations were performed to investigate the mechanism of adsorption. Considering potential hydrogen bond interaction between flavonols and collagen, the 11-mer sequence Ace-Gly-Pro-Hyp-Gly-Ala-Ser-Gly-Glu-Arg-Nm was selected as the collagen- like peptide model $^{17}$ for its rich hydrogen bonding sites on side groups and relative availability of actual collagen molecule. Molecular docking and molecular dynamics investigation have been carried out to gain insight into the affinity of collagen-like peptide with myricetin, quercetin and kaempferol under the condition of water and ethanol respectively.

\section{EXPERIMENTAL}

Kaempfrol, quercetin and myricetin were purchased from Hui Ke Botanical Research and Development Corp. Ltd, China. The purity of the components was $\geq 95.0 \%$. All reagents were of analytical grade.

UV analysis of kaempferol, quercetin and myricetin: Ultraviolet visible light spectrophotometer (UV751GD, Perkin-Elmer, Ltd., USA) was used to analyze the concentration of the components in solutions. The wavelength of kampferol, quercetin and myricetin were $360 \mathrm{~nm}$.

Adsorption isotherms in ethanol solution: The glutaraldehyde cross-linked collagen fiber (GCF) was employed to study the static emperiment. The GCF was prepared according to the process described in our previous work ${ }^{18}$. For the solubility of the three flavonols are very little in the pure water and they should be dissolved in the ethanol solution, so the experiments of adsorption isotherms were conducted in pure ethanol. The initial concentrations of kaempferol or quercetin or myricetin were $0.5-4.8 \mathrm{mg} / \mathrm{mL}$ at 288,298 and $308 \mathrm{~K}$, respectively. $0.15 \mathrm{~g}$ of GCF was weighted into each $50 \mathrm{~mL}$ flask, to which $15 \mathrm{~mL}$ each flavonols solution was added. Adsorption equilibrium achieved after $6 \mathrm{~h}$ oscillation under the condition of 120 rotations per minute.

The amount of the three flavonols per gram of adsorbent $\mathrm{q}(\mathrm{mmol} / \mathrm{g})$ was calculated as:

$$
\mathrm{q}=\mathrm{V}\left(\mathrm{C}-\mathrm{C}_{\mathrm{e}}\right) / \mathrm{W}
$$

where $\mathrm{C}$ and $\mathrm{C}_{\mathrm{e}}$ are the initial and equilibrium solute concentrations $(\mathrm{mmol} / \mathrm{L}), \mathrm{V}$ is the solution volume $(\mathrm{L})$ and $\mathrm{W}$ is the weight of the adsorbent $(\mathrm{g})$.

The adsorption affinities $\left(q / C_{e}\right)$ were determined from the slopes of individual isotherms in the linear region and the values are identical to the averaged values of individual adsorption affinities ${ }^{16}$.

All simulations were performed by using the software package Accelry Discovery Studio 2.5 (Accelrys Inc., San Diego, 2001-2009) on a DELL computer. All calculations are derived from the CHARMm force field.

The model 11-mer collagen-like peptide was built by using Biopolymer module and the value of $(\theta, \varphi)$ should be in Ramachandran plot. The structures of myricetin, quercetin, kaempferol were generated by using Builder module and were refined through energy minimization. Molecular docking was carried out to identify the binding sites of collgen-like peptide with myricetin, quercetin, kaempferol respectively by using CDOCKER module, then a series of complexes and the corresponding CDOCKER energies could be obtained. For each favonol only one complex was selected to do molecular dynamic simulation to see the time evolution of the complex by using Discover module. Molecular dynamic simulations were performed at the canonical (NVT) ensemble and the time step of the integration was held on $1 \mathrm{fs}$. The geometry of complexes was optimized by steepest descents algorith method of 2000 iterations followed by conjugate gradients algorithm. The NVT molecular dynamic simulation was performed a production period of 5200 ps including an equilibration period of $200 \mathrm{ps}$ at $300 \mathrm{~K}$. In order to mimic the medium effect on the interaction between peptide and flavonols, implicit solvent model was used and the scalar dielectric constant of 78.5 or 24.5 , corresponding to water or ethanol, was applied each complex, respectively.

Molecular docking and binding energy: Molecular docking is a method which can predict the preferred orientation of one molecule to the other to form complex ${ }^{19}$, which is frequently used to predict the affinity and activity of the small molecule in the process of discovery of new medicine ${ }^{20}$. The three flavonols were non-covalently docked with the collagenlike peptide using flexible docking respctively. For each final pose, the CHARMm energy (interaction energy plus ligand strain energy) and the interaction energy alone are calculated. The CDOCKER score reported as the negative value (i.e., CDOCKER energy), where a lower value indicate a more favourable binding ${ }^{21}$. From the docking analysis, the CDOCKER energies value against pose number could be obtained. Then for each flavonol, the complexes with higher scoring would be listed and the highest was selected to study the binding energy and behaviour of molecular dynamics.

After docking, the binding energy between the collagenlike peptide and three flavonols in water and ethanol is calculated further to investigate the solvation effects on the affinity of the polyphenols bonding with collagen, the binding energy exhibits significant relevance to the experimental binding ${ }^{22}$ and is calculated based on the following equation ${ }^{21}$ : Binding energy $=$ Total energy of complex - (Total energy of peptide + Total energy of flavonol) 
Then, based on the equation, the higher binding energy indicates weaker interaction between collagen-like peptide and favonol.

Analysis of the trajectories: Molecular dynamics (MD) simulations aimed to check the temporal stability of the flavonol-peptide complexes. The root mean square deviation (RMSD) was computed for the peptide backbone atoms referring to the starting structures of the molecular dynamics simulation. The time evolution of potential energy of complex was also achieved in water and ethanol respectively.

Then, hydrogen bond analysis was done based on the conformations of the last $2 \mathrm{~ns}$ from the trajectories. Hydrogen bonds (DH...A)were calculated and visualized by using the $\mathrm{H}$-bond facility in the Viewer module. The criterions of (D...A) distance $\leq 0.25 \mathrm{~nm}$ and the bond angle (D-H...A) $>130^{\circ}$ were chosen $^{23,24}$. From the number of hydrogen bond, it can make clear the contribution of hydrogen bond to the mode of interaction between peptide and the three flavonols in different solvents.

The radial distribution function (RDF) is a useful parameter to describe the distribution of flavonols around collagenlike peptide under two different implicit solvents (water and ethanol). The radial distribution function was calculated from the trajectory based on the peptide or amino acid residue with the hydroxylic oxygen atoms of flavonols.

Interaction energies of collegen-like peptide with myricetin, quercetin, kaempferol, representing the contribution of non-bonded interaction in the CHARMm potential function were calculated based on the trajectory. Interaction energy has two components, electrostatic interaction energy and van der Waals interaction energy ${ }^{21}$. It can directly show the main non-bonded affinity of collagen-like peptide with myricetin, quercetin, kaempferol.

\section{RESULTS AND DISCUSSION}

Static experiments: The adsorption affinity $\left(q / C_{e}\right)$ is defined as the equilibrium ratio of the solid-phase concentration (mmol/g sorbent) to the liquid-phase solute concentration $(\mathrm{mmol} / \mathrm{L})$. The adsorption affinities for each of the flavonols are calculated in Table- 1 .
TABLE-1

ADSORPTION AFFINITY FOR THREE FLAVONOLS ONTO GCF

\begin{tabular}{cc}
\hline Adsorbate & Adsorption affinity $\mathrm{q} / \mathrm{C}_{\mathrm{e}}(\mathrm{L} / \mathrm{g})$ \\
\hline Kaempferol & 0.023 \\
Quercetin & 0.029 \\
Myricetin & 0.034 \\
\hline
\end{tabular}

From Table-1, myricetin exhibits the highest adsorption affinity to GCF while kaempferol has the lowest adsorption affinity. The adsorption capacities of the flavonols on GCF obey the following order: myricetin $>$ quercetin $>$ kaempferol. The GCF showed different affinity with flavonols from the static experiments. Then, it is interesting to explore the mechanism of three flavonols on collagen fiber.

Complexes of collagen-like peptide with the three flavonols: The hydroxyl, carboxyl and basic group in the side chain of collagen-like peptide has been considered as a hydrogen bond donor or acceptor for hydroxyl or carbonyl of myricetin, quercetin and kaempferol and for each target 50 most favourable poses were retained. The binding sites and CDOCKER energies of complexes formed by hydroxyproline (Hyp), serine (Ser), glutamic acid (Glu) and arginine (Arg) of collagen-like peptide with three flavonols were listed in Table- 2 and for each flavonol only the complex with highest interaction energy was depicted (Fig. 2). The backbone $\mathrm{C}=\mathrm{O}$ and $\mathrm{N}-\mathrm{H}$ groups of the peptide sequence have not been considerd, because these groups are involved in the intra-chain hydrogen bonding in the actual collagen triple helical molecule $^{20}$. Indeed, the interaction energies of complex formed by backbone $\mathrm{C}=\mathrm{O}$ or $\mathrm{N}-\mathrm{H}$ groups with flavonols were much lower compared with those of functional groups of side chain from our docking.

Arginine is the favourable site to dock with the three flavonols based on the CDOCKER energy from Table-2. The CDOCKER energies of the complexes $1,4,8$ are -14.4568 $\mathrm{kcal} / \mathrm{mol},-13.9351 \mathrm{kcal} / \mathrm{mol},-9.5386 \mathrm{kcal} / \mathrm{mol}$, respectively, lower than those of other sites with corresponding flavonol. The poses with lower CDOCKER energy occur arginine binding with $\mathrm{OH}$ in the $\mathrm{B}$ ring of myricetin, quercetin and kaempferol from the detail of $\mathrm{H}$-bond listed in Table-2. It could be inferred that the number and position of hydroxyl group in

TABLE-2

CDOCKER ENERGY OF COLLAGEN-LIKE PEPTIDE WITH MYRICETIN, QUERCETIN AND KAEMPFEROL

\begin{tabular}{|c|c|c|c|}
\hline Complex number & $\begin{array}{l}\text { Complex (small molecular: amino } \\
\text { acid of the collagen-like peptide) }\end{array}$ & H-bond details & CDOCKER energy $(\mathrm{kcal} / \mathrm{mol})$ \\
\hline 1 & Myricetin:Arg- $\mathrm{NH}_{2}$ & $* \mathrm{~B}-m-(\mathrm{H}) \mathrm{O}: \mathrm{H}_{2} \mathrm{~N}-\mathrm{Arg}$ & -14.4568 \\
\hline \multirow[t]{2}{*}{2} & Myricetin:Glu-COOH & B-p-OH: $\mathrm{O}=\mathrm{C}(\mathrm{COOH})-\mathrm{Glu}$ & -13.5317 \\
\hline & Myricetin:Ser-OH & C-(C-O-C)-O:HO-Ser & \\
\hline 3 & Myricetin:Hyp-OH & C-(C-O-C)-O:HO-Hyp & -8.8737 \\
\hline 4 & Quercetin:Arg-NH & B- $m(\mathrm{H}) \mathrm{O}: \mathrm{H}_{2} \mathrm{~N}-\mathrm{Arg}$ & -13.9351 \\
\hline 5 & Quercetin:Glu-COOH & $\begin{array}{l}\mathrm{B}-m-(\mathrm{O}) \mathrm{H}: \mathrm{O}=\mathrm{C}(\mathrm{COOH})-\mathrm{Glu} \\
\mathrm{B}-m-(\mathrm{O}) \mathrm{H}: \mathrm{OH}(\mathrm{COOH})-\mathrm{Glu}\end{array}$ & -12.4264 \\
\hline 6 & Quercetin:Ser-OH & A-o-(H)O:HO-Ser & -12.7378 \\
\hline 7 & Quercetin: Hyp-OH & $\mathrm{C}-\mathrm{C}=\mathrm{O}: \mathrm{HO}-\mathrm{Hyp}$ & -7.6585 \\
\hline 8 & Kaempferol:Glu-COOH & B-p-(O)H: OH(COOH)-Glu & -9.5386 \\
\hline 9 & Kaempferol:Arg-NH/ $\mathrm{NH}_{2}$ & $\begin{array}{l}\mathrm{C}-\mathrm{C}=\mathrm{O}: \mathrm{HN}-\mathrm{Arg} \\
\mathrm{C}-\mathrm{C}=\mathrm{O}: \mathrm{H}_{2} \mathrm{~N}-\mathrm{Arg}\end{array}$ & -7.5575 \\
\hline 10 & Kaempferol:Ser-OH & $\mathrm{C}-(\mathrm{C}-\mathrm{O}-\mathrm{C})-\mathrm{O}: \mathrm{HO}-\mathrm{Ser}$ & -8.1089 \\
\hline 11 & Kaempferol: Hyp-OH & C-(C-O-C)-O: HO-Hyp & -4.6301 \\
\hline
\end{tabular}

$* \mathrm{~A}, \mathrm{~B}, \mathrm{C}$ represent the three rings of the flavonol respectively; $p, m, o$ describe para-position, meta- position, ortho-position of the ring. 


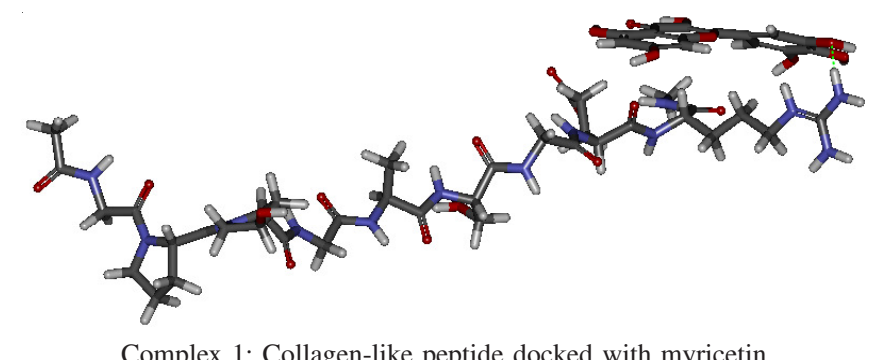

Complex 1: Collagen-like peptide docked with myricetin

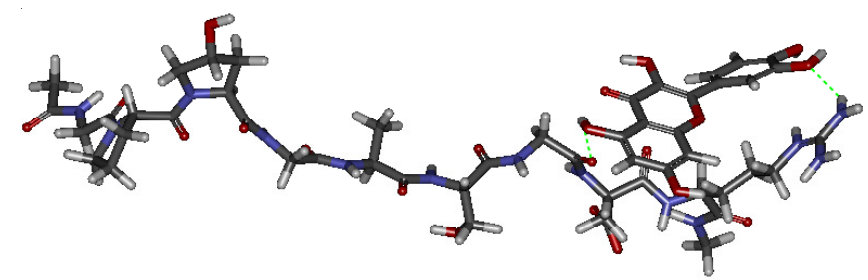

Complex 4: Collagen-like peptide docked with quercetin

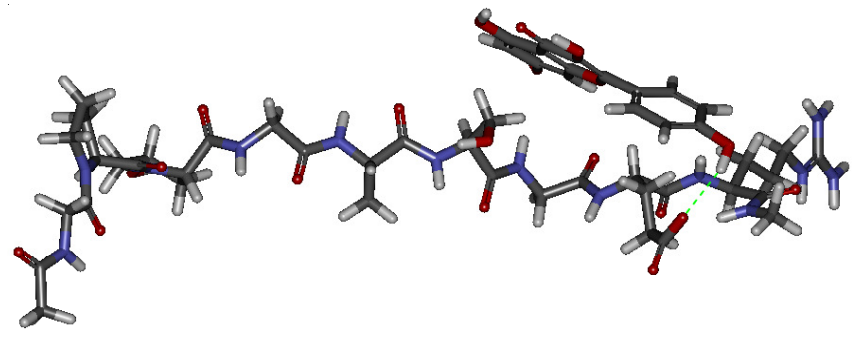

Complex 8: Collagen-like peptide docked with kaempferol

Fig. 2. Docking results of myricetin, quercetin, kaempferol with collagen

B ring of favonols would influence the docking energy and affinity. Because myricetin has three hydroxyls (two meta$\mathrm{OH}$ and one para-OH) in B ring, its docking energy is lower than those of quercetin and kaempferol.

Solvent effects on the binding energy of three flavonols with collagen-like peptide: It has shown some differences among the complexes of the flavonols with collagen from docking interaction energy. In order to further identify effective separation of the three flavonols by collagen, binding energy of the ligand-receptor in different solvents was calculated. The complexes 1, 4, 8 in Table-2 were selected to calculate binding energy in water and ethanol by using solvents dielectric constants 78.5 and 24.5 module, respectively. The results are shown in Table-3.

TABLE-3

BINDING ENERGY OF THE LIGAND-RECEPTOR IN WATER AND ETHANOL SOLVENTS

\begin{tabular}{clcc}
\hline $\begin{array}{c}\text { Complex } \\
\text { number }\end{array}$ & $\begin{array}{c}\text { Complex (small } \\
\text { molecular: amino acid of } \\
\text { the collagen-like peptide) }\end{array}$ & $\begin{array}{c}\text { Binding } \\
\text { energy in } \\
\text { water } \\
(\mathrm{kcal} / \mathrm{mol})\end{array}$ & $\begin{array}{c}\text { Binding } \\
\text { energy in } \\
\text { ethanol } \\
(\mathrm{kcal} / \mathrm{mol})\end{array}$ \\
\hline 1 & Myricetin:Arg-NH & -15.3538 & -19.7418 \\
4 & Quercetin:Arg-NH & -15.6471 & -16.3349 \\
8 & Kaempferol:Glu-COOH & -13.9786 & -14.6369 \\
\hline
\end{tabular}

The binding energy of kaempferol-collagen complex is the highest in both water and ethanol solvent, indicating weak interaction between kaempferol and collagen. From Table-3, the interaction of collagen-like peptide with three flavonols in ethanol was stronger than that in water based on the lower binding energy. In water, the binding energy is similar for the three complexes and the difference is not obvious. For example, the difference of binding energy between complex myricetincollagen and quercetin-collagen is very slight, about $0.3 \mathrm{kcal} /$ mol. However, in ethanol the difference is magnified 11 times, about $3.3 \mathrm{kcal} / \mathrm{mol}$. The result indicates that the solvent could change the affinity of flavonols with collagen. Even more important, solvent could magnify the difference of affinity, which has certain guiding significance in the experiment separation process.

Solvent effects on structural stability of the three complexes: Molecular dynamics simulations have been carried out on the energy-minimized complex 1, 4 and 8 in water and ethanol, respectively. The potential energy map of the three complexes in water was shown in Fig. 3. It could be deduced that these systems were stable at $300 \mathrm{~K}$ and were sufficiently equilibrated from the slight energy fluctuation given in Fig. 3. The potential energy of complex 1, 4 and 8 was found to be stable at $126.4 \mathrm{kal} / \mathrm{mol}, 121.7 \mathrm{kcal} / \mathrm{mol}$ and $120.7 \mathrm{kcal} / \mathrm{mol}$ in water and $116.7 \mathrm{kcal} / \mathrm{mol}, 117.9 \mathrm{kcal} / \mathrm{mol}$ and $118.4 \mathrm{kcal} / \mathrm{mol}$ in ethanol, respectively.

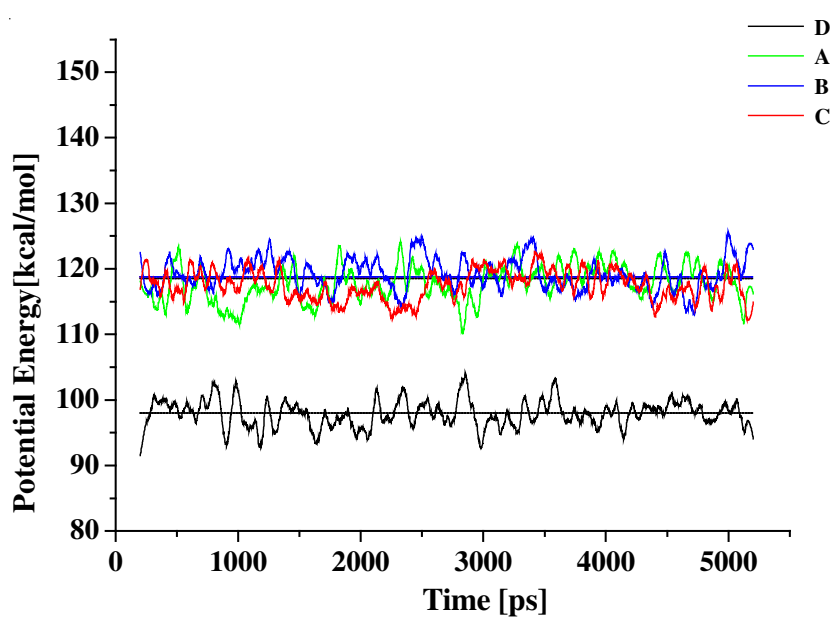

Fig. 3. Potential energy values of the complex A, B, C, D; A: peptide with myricetin; B: peptide with quercetin; C: peptide with kaempferol; D: peptide

The conformationl structural stabilities of the three complexes (collagen-like peptide combining with myricetin, quercetin or kaempferol) are analyzed by the root-mean-square deviation (RMSD) of backbone atoms from the starting structure. The behaviour of RMSD as a function of time under different dielectric constants is presented in Figs. 4 and 5. In water, the RMSD average value of complex 1 is the lowest, $6.03 \AA$ and the average values of complex 4 and 8 are $10.69 \AA$ and 11.07 $\AA$, respectively. The least root-mean-square deviation of complex 1 indicates myricetin could make the peptide structure more stable than quercetin or kaempferol. The structural modification of collagen-like peptide as a consequence of the interaction with three flavonols is in accordance with experimental findings obtained by differential scanning calorimetry (DSC), showing that the interaction with bayberry tannin (containing O-trihydroxyl just like myricetin) increases the hydrothermal stability of collagen much more than with Larch tannin (containing Odihydroxyl like quercetin $)^{25,26}$. 


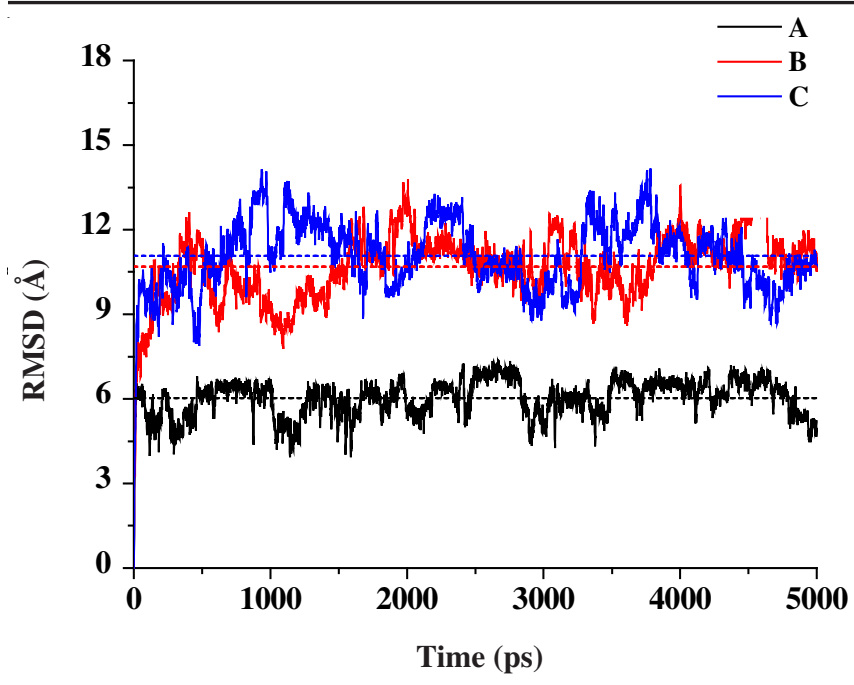

Fig. 4. Backbone RMSD values of complex A, B, C in water; A: peptide with myricetin; B: peptide with quercetin; C: peptide with kaempferol

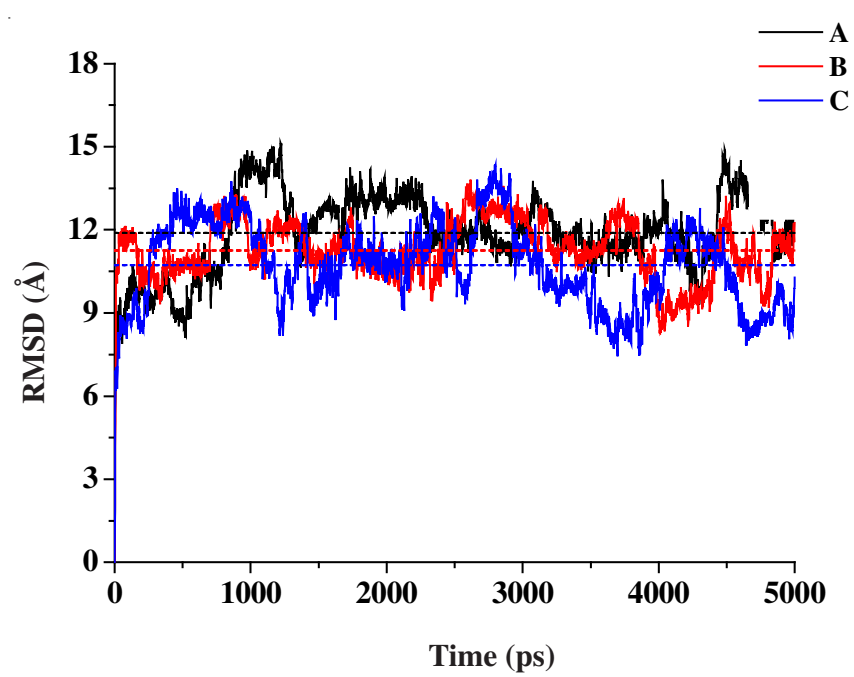

Fig. 5. Backbone RMSD values of complex A, B, C; A: peptide with myricetin; B: peptide with quercetin; C: peptide with kaempferol

It is obvious that solvent has greater effect on the dynamic behaviour of complex 1 formed by collagen-like peptide and myricetin. The RMSD average value of complex 1 increases from $6.03 \AA$ in water to $11.89 \AA$ in ethanol. The deviations suggest major changes in stability of collagen-like peptide by the interaction with flavonols in different solvents, which is consistent with the result of binding energy. The behavors of complex 4 and 8 also change more or less in water and ethanol. Small differences in dynamic behaviour can be linked to affinity differences, furthermore, directly related to structural differences of flavonols ${ }^{27}$. It could be inferred that if the solvent is changed, the structure stability of complex will be changed.

H-bond analysis: To keep track of the potential hydrogen bond interactions, the distribution of flavonols around the collagen-like peptide was evaluated by the radial distribution function (RDF). Radial distribution function was calculated from the trajectory based on the peptide or amino acid residue with the hydroxylic oxygen atoms of flavonols. In Fig. 6, the RDFs exhibit large peaks with peak position in the range 4.5-
$5.5 \AA$ and the peaks in water are higher than those in ethanol for the three complexes. It indicates that solvent would change the distribution of flavonols around peptide and then affect the formation of inter-molecular H-bond. In addition, the peak position at such a distance is not favour of forming intermolecular H-bond. The RDF of peptide with phenolic-hydroxyl of quercetin is much higher than the others. May be the steric effect and intra-molecular H-bond of myricetin would hinder the formation of intermolecular H-bond with peptide ${ }^{28}$.
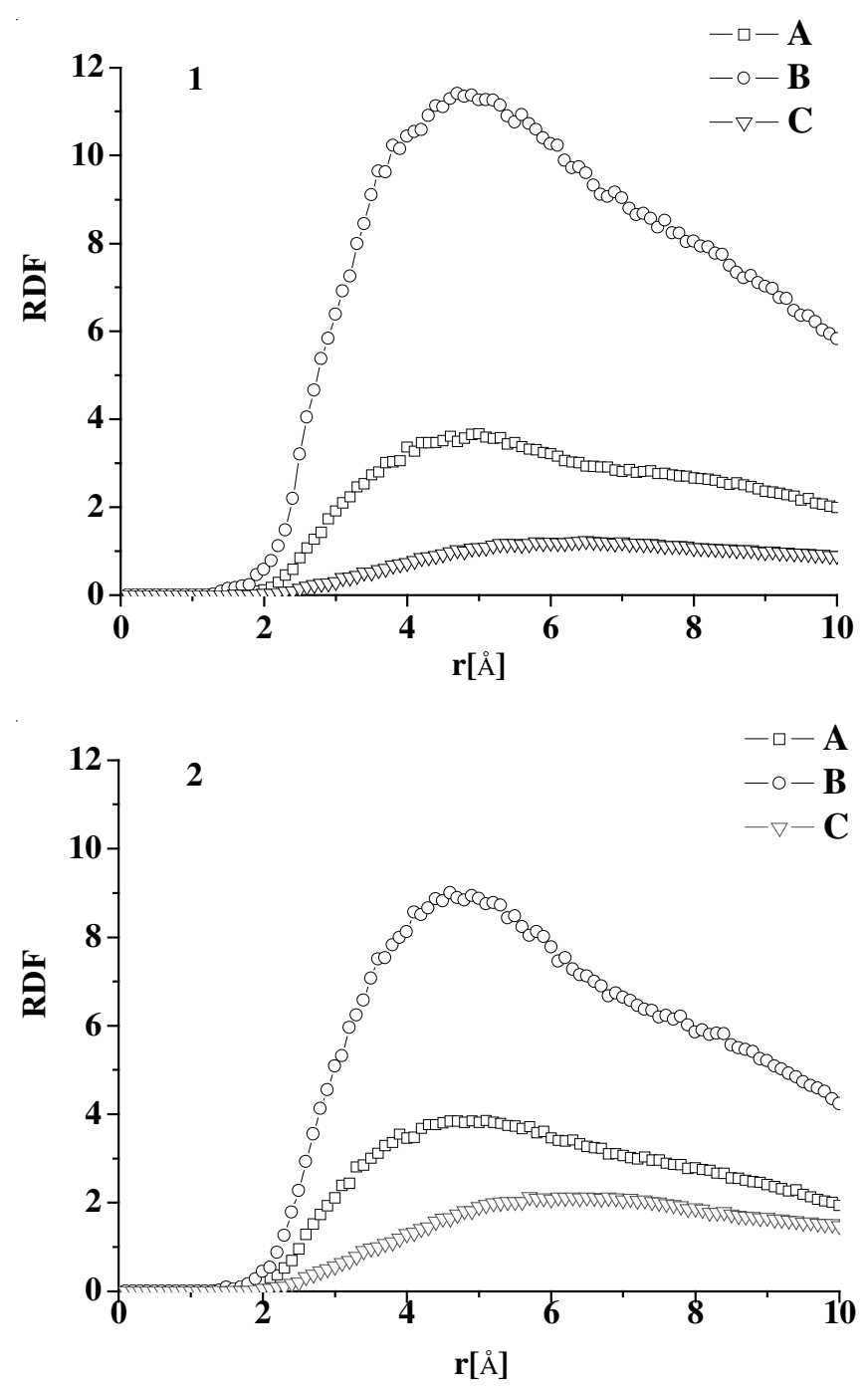

Fig. 6. Radial distribution function of peptide with phenolic-hydroxyl of flavonols (A: myricetin, B: quercetin, C: kaempferol) in water (1) and ethanol (2)

To identify flavonol molecules preferably binding to amino acid residues of peptide, the partial radical distribution functions (prdfs) of the hydroxylic oxygen of flavonols with various amino acid of peptide are shown in Fig. 7. Obviously, Hyp and Ser are favourite sites of flavonols to bind from the peak height of partial radical distribution function. The solvent could affect the kind of amino acid which flavonols favour to interact. For example, in water myricetin interact more favourably with Hyp and Glu than with Arg and Ser. However in ethanol, Ser is the favourite from Fig. $7 \mathrm{~A}$ and $\mathrm{A}^{\prime}$. The solvent also changes the height of peak, indicating the ability to change H-bonding network between flavonols and amino acid residues. 

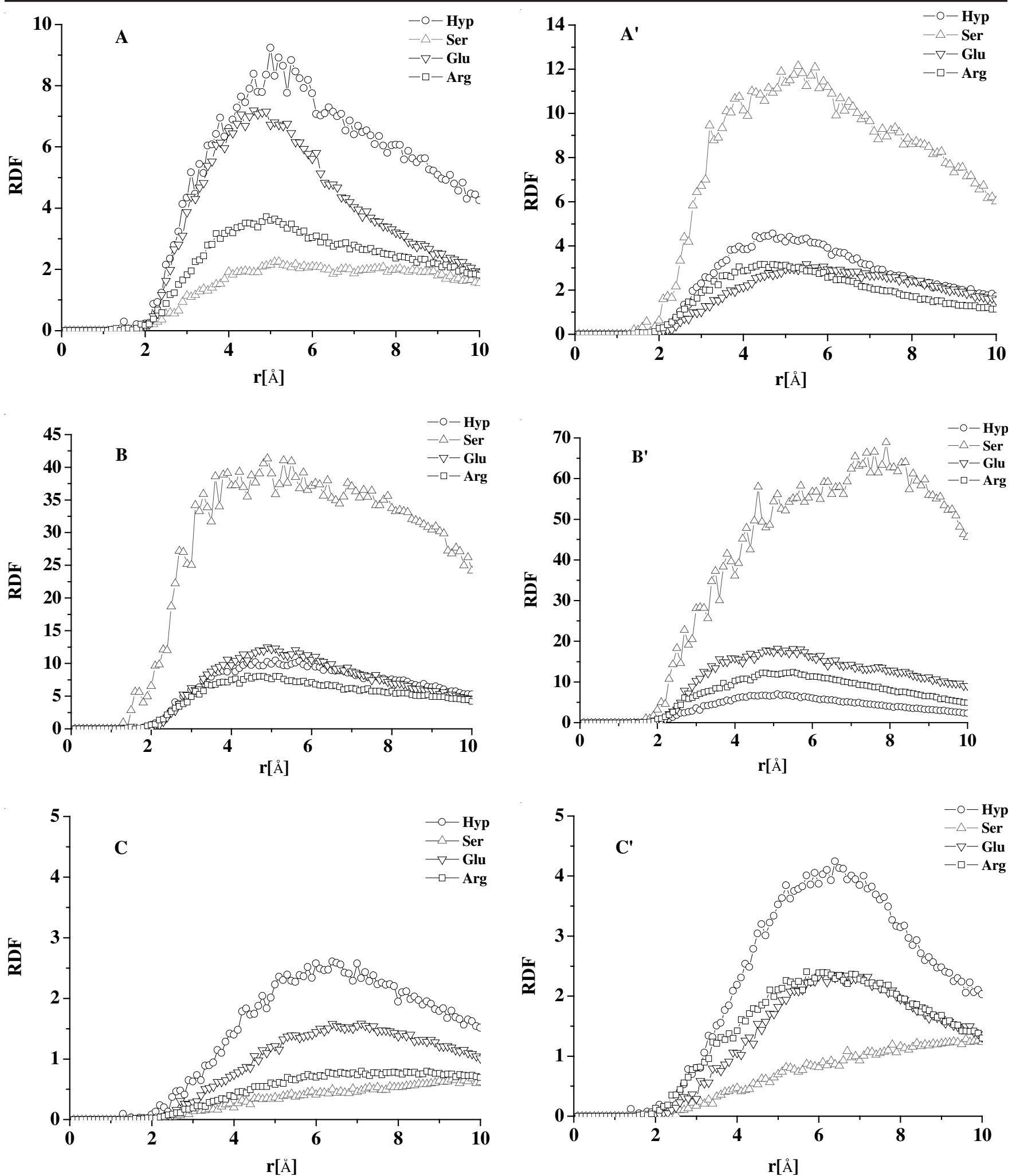

Fig. 7. PRDF for amino acid residue of peptide with phenolic-hydroxyl of flavonols: A, B, C are respectively myricetin, quercetin, kaempferol in water; $\mathrm{A}^{\prime}, \mathrm{B}^{\prime}, \mathrm{C}^{\prime}$ are respectively myricetin, quercetin, kaempferol in ethanol

To give a clear picture of the hydrogen bonding network between flavonols and collagen-like peptide residues, the number $\left(\mathrm{n}_{\mathrm{H}}\right)$ and fraction of conformations $\left(\mathrm{f}_{\mathrm{H}}\right)$ of each flavonol to form a H-bond with amino residues are counted and calculated over the last $2 \mathrm{~ns}$ and the fraction is obtained from the following equation:

$$
\mathrm{f}_{\mathrm{H}}=\frac{\mathrm{n}_{\mathrm{H}}}{\mathrm{N}} \times 100 \%
$$

$\mathrm{N}$ is the total conformations of trajectory sampled. There are total 2000 conformations over the last 2 ns dynamics simulated system. The H-bond numbers over the last $2 \mathrm{~ns}$ of the three complexes are presented in Table-4. 


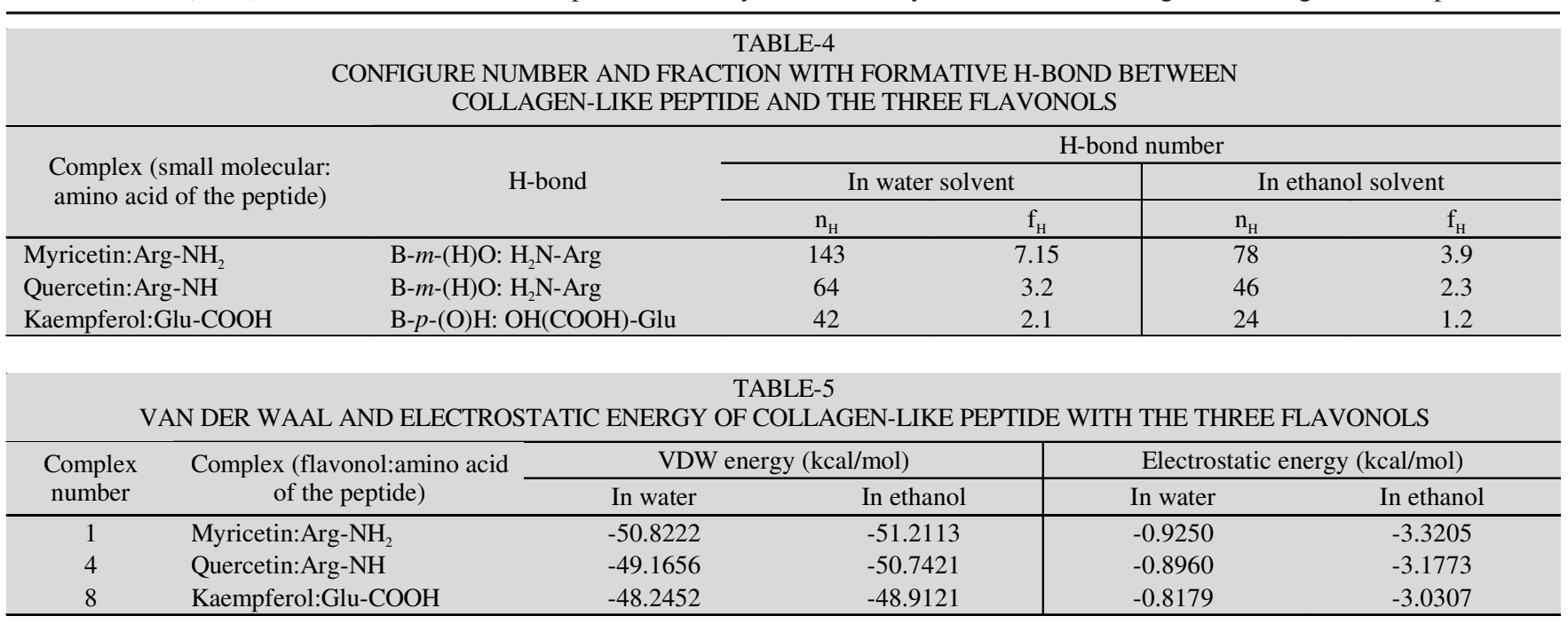

The number of configures with formative H-bond is low relative to 2000 conformations and the solvent has great effect on the number. The inter-molecular H-bond has a decreasing tendency changing solvent from water to ethanol. The configure fraction number of $\mathrm{H}$-bond formed by peptide with flavonols in water is almost 2 times of that in ethanol, indicating the effect of solvent on stability of $\mathrm{H}$-bond between collagen-like peptide and flavonols. For myricetin, the number decreases gradually from 143 in water to 78 in ethanol. From Table-4, the maximum $\mathrm{H}$-bond ratio is $7.15 \%$, which occurs in myricetin bonding with collagen-like peptide, suggesting that the number of hydroxyl in B ring of flavonols would affect the stability of intermolecular H-bond formed.

Component of the interaction energy of collagen-like peptide with flavonols: The mechanism of collagen-like peptide in separating the three flavonols is examined in more detail by analyzing the interaction energy components between peptide and myricetin, quercetin, as well as kaempferol respectively. Calculation of the peptide-flavonol interaction energies was carried out by using a two-term potential function (van der Waals and electrostatic) with geometric and energy parameters derived from the force field of CHARMm. From Table-5, the main component of the interaction energy of myricetin, quercetin, kaempferol bonding with collagen-like peptide is the van der waals term under the condition of both water and ethanol. The polarity of solvent affect the value of interaction energy and in ethanol both the van der Waals and electrostatic components increase. The electrostatic energy increases at least $2 \mathrm{kcal} / \mathrm{mol}$ in ethanol than in water.

Previous experiments found the dominant mode of interaction between polyphenols and collagen fibers is a synergistic effect of hydrophobic association and hydrogen bonding ${ }^{29}$. The result obtained from the analysis of $\mathrm{H}$-bond and interaction energy provided further evidence that solvent could change the mode of interaction to change the affinity of collagen-like peptide with flavonols. This would be helpful to the experimental study and application of collagen-related materials to isolate the three flavonols.

\section{Conclusion}

In this research, the adsorption isotherms of the glutaraldehyde cross-linked collagen fiber (GCF) to myricetin, quercetin and kaempferol were obtained from static experiment. Then the affinity and essential difference of myricetin, quercetin, kaempferol bonding with collagen-like peptide in two solvents are analyzed by molecular docking, calculating binding energy and molecular dynamic simulation. The adsorption capacities of the flavonols on GCF follow the order: myricetin > quercetin $>$ kaempferol. Molecular simulation showed the most stable docking energies of the peptide with myricetin, quercetin, kaempferol are $-14.4568 \mathrm{kcal} / \mathrm{mol},-13.9351 \mathrm{kcal} / \mathrm{mol}$ and $-9.53861 \mathrm{kcal} / \mathrm{mol}$, respectively, which is consistent with the experimental results. Solvent can change the binding energy of collagen-like peptide with flavonols and magnify the difference of binding energy among the three flavonols. Molecular dynamics simulation indicates great differences in the behaviour of complexes of peptide with myricetin, quercetin and kaempferol under water and ethanol solvents based on RMSD. The analysis of trajetory shows further that the main affinities of myricetin, quercetin, kaempferol bonding with collagen-like peptide are from van der waals' force (VDW) and H-bonds, whereas electrostatic interaction contributes much less. Furthermore, solvent could change the contribution of H-bond, van der Waal and electrostatic interaction to the mode of interaction between peptide and flavonols in different ways. So it can infer, in practice, if the ratio of water and ethanol is proper to be adjusted, the three flavonols (myricetin, quercetin, kaempferol) may be isolated.

\section{ACKNOWLEDGEMENTS}

The research grant in part is funded by National Natural Science Foundation of China (21076130) and the Program for New Century Excellent (NCET-11-0358).

\section{REFERENCES}

1. S. Karakaya and S.N. EL, Food Chem., 66, 289 (1999).

2. A.L. Catapano, Angiology, 48, 39 (1997).

3. M.G.L. Hertog, P.C.H. Hollman and B. Putte, J. Agric. Food Chem., 41, 1242 (1993).

4. M.G.L. Hertog, E.J.M. Feskens, P.C.H. Hollman, M.B. Katan and D. Kromhout, The Zutphen Elderly Study, 342, 1007 (1993).

5. P.C.H. Hollman, M.G.L. Hertog and M.B. Katan, Food Chem., 57, 43 (1996).

6. Z.X. Zhong, X.F. Chen and G.F. Zhou, Chin. J. Modern Appl. Pharm., 20, 466 (2003). 
7. B.X. Zang, M. Jin and W. Wu, Acta Pharmaceut. Sin., 38, 831 (2003).

8. P.C.H. Hollman, J.H.M. de Vries and M.B. Katan, Atherosclerosis, 115 S49 (1995).

9. W.Z. Zhang, X. Zhou and X.Z. Zhao, Chin. J. Misdiagnostics, 7, 5219 (2007)

10. H. Seibert, E. Maser, K. Schweda, S. Seibert and M. Gulden, Food Chem. Toxicol., 49, 2398 (2011).

11. J. Li, X.P. Liao, G.H. Liao, Q. He, W.H. Zhang and B. Shi, J. Sep. Sci., 33, 2230 (2010).

12. B. Shi, J. Am. Leather Chem. Assoc., 103, 270 (2008).

13. A.J. Charlton, N.J. Baxter and M.L. Khan, J. Agric. Food Chem., 50, 1593 (2002)

14. C. Giudici and M. Viola, FEBS Lett., 547, 170 (2003).

15. S. Bronco, C. Cappelli and S. Monti, J. Phys. Chem. B, 108, 10101 (2004).

16. P. Gogoi, N.N. Dutta and P.G. Rao, Biochem. Eng. J., 52, 144 (2010).

17. B. Madhan, Thanikaivelan, V. Subramanian and J.R. Rao, Chem. Phys. Lett., 346, 334 (2001).
18. X.P. Liao and B. Shi, Sci. Food Agric., 85, 1285 (2005).

19. T. Lengauer and M. Rarey, Curr. Opin. Struct. Biol., 6, 402 (1996).

20. D.B. Kitchen, H. Decornez, J.R. Furr and J. Bajorath, Nat. Rev. Drug Discov., 3, 935 (2004).

21. Help Topics of Accelry Discovery Studio 2.5 (Accelrys Inc., San Diego, 2001-2009).

22. X. Chen, Z.L. Ji and D.G. Zhi, Computers Chem., 26, 661 (2002).

23. T. Steiner, Angew. Chem. Int. Ed., 41, 48 (2002).

24. G.F. Fabiola, V. Nagarajan and V. Pattabhi, Acta Crystallogr., 53, 316 (1997).

25. Z.-B. Lu and X.P. Liao, Chem. Ind. Forest Prod., 24, 7 (2004).

26. W.Y. Chen and G.Y. Li, Tanning Chemical, China Light Industry Press, 99-107 (2005).

27. R. Gil-Redondo, J. Klett and A. Morreale, Proteins, 78, 162 (2010).

28. W.H. Zhang and B. Shi, J. Soc. Leather Technol. Chem., 89, 137 (2005).

29. X.P. Liao, Z.B. Lu and B. Shi, Ind. Eng. Chem. Res., 42, 3397 (2003). 